\title{
Ergodicity of mapping class group actions on representation varieties, I. Closed surfaces
}

Doug Pickrell and Eugene Z. Xia

\begin{abstract}
We prove that the mapping class group of a closed surface acts ergodically on connected components of the representation variety corresponding to a connected compact Lie group.
\end{abstract}

Mathematics Subject Classification (2000). 22F50.

Keywords. Fundamental group of surface, compact Lie group, moduli space, mapping class group, ergodic theory.

\section{Introduction}

Throughout this paper we fix a connected compact Lie group $K$, and we let $d g$ denote the unique normalized Haar measure on $K$.

Let $\Sigma$ denote a closed oriented surface with a fixed basepoint, and let $\Gamma_{\Sigma}=$ $\pi_{0}(\operatorname{Aut}(\Sigma))$, the mapping class group. The representation variety $\operatorname{Hom}\left(\pi_{1} \Sigma, K\right)$ has a canonical $\Gamma_{\Sigma}$-invariant measure class, the Lebesgue class of the set of nonsingular points. Our aim is to prove the following

Theorem (0.1). The group $\Gamma_{\Sigma}$ acts ergodically on the Lebesgue class of each connected component of $\operatorname{Hom}\left(\pi_{1} \Sigma, K\right)$.

Let $H^{1}(\Sigma, K)$ denote the moduli space of representations, i.e. the quotient of $\operatorname{Hom}\left(\pi_{1} \Sigma, K\right)$ by the conjugation action of $K$. It is well-known that this moduli space has a $\Gamma_{\Sigma}$-invariant symplectic structure ([AB], [Go1]). The following was proven by Goldman for $K$ locally isomorphic to a product of $S U(2)$ 's and a torus, in [Go2].

Corollary (0.2). The group $\Gamma_{\Sigma}$ acts ergodically on the Lebesgue class of each connected component of $H^{1}(\Sigma, K)$. Thus the Lebesgue class of each component has an essentially unique $\Gamma_{\Sigma}$-invariant representative, the canonical symplectic volume element. 
In broad outline our basic idea is to prove an analogue of (0.1) for one and two-holed tori, for a.e. boundary condition; this is easier than dealing with every boundary condition, because we can use harmonic analysis for $K$. When we sew to obtain more complicated surfaces, because we integrate, the measure-theoretic ambiguity is washed away. For the special cases that we consider, the question of ergodicity for the discrete mapping class group is in fact equivalent to ergodicity for a much larger group. This observation is useful in a number of contexts, and to display the idea as clearly as possible we have chosen to concentrate on the closed surface case in this paper.

Our Theorem is of interest for a number of reasons, some of which are discussed in [Go2]. Our own original motivations involved quantum field theory, which incidentally explains the nature of our methods. A first point of contact with QFT is the 2-dimensional Yang-Mills measure $d \nu_{T^{-1} Y M_{2}}$ on the space of gauge equivalence classes of all (generalized) $K$-connections (see $[\mathrm{Pi}]$ and the references there). Corollary (0.2) a priori determines the form of the classical $T \downarrow 0$ limit, which is determined by direct calculation in [Fo]. Elsewhere we will prove that the Yang-Mills measures are ergodic with respect to their symmetry group, the group of area-preserving diffeomorphisms, essentially using the methods established in this paper.

A second point of contact involves Chern-Simons gauge theory, for which the moduli space $H^{1}(\Sigma, K)$ is the classical phase space. It is known that the action of $\Gamma_{\Sigma}$ on the quantum state space is reducible for arbitrarily high levels (see $\$ 17.1 .3$ of $[\mathrm{DMS}]$ ). Thus we have interesting examples of a group acting ergodically on a compact phase space and reducibly on the corresponding quantization, even in the classical limit.

For results on the topological dynamics of $\Gamma_{\Sigma}$ acting on the moduli space, see $[\mathrm{PX}]$.

Notation (0.2). Given a Lie group $G$, we will always use left translation to trivialize the tangent bundle:

$$
T G \rightarrow G \times \mathfrak{g}:\left.v\right|_{g} \rightarrow\left(L_{g^{-1}}\right)_{*}\left(\left.v\right|_{g}\right)
$$

In this frame the commutator of two vector fields $x, y: G \rightarrow \mathfrak{g}$ is given by

$$
\left.[x, y]\right|_{g}=\left.d y(x)\right|_{g}-\left.d x(y)\right|_{g}+[x(g), y(g)] .
$$

The adjoint action $\operatorname{Ad}: G \times \mathfrak{g} \rightarrow \mathfrak{g}$ is abbreviated to $\operatorname{Ad}_{g}(x)=x^{g}$. If $G$ acts on a space $X$, then $X^{g}$ denotes the fixed point set.

Acknowledgment. During the course of this research, Eugene Z. Xia was with the University of Arizona. 


\section{Basic notions and sewing}

For the purposes of this paper, we will need to consider a somewhat nonstandard kind of boundary condition for surfaces with boundary.

Consider a connected compact oriented surface $\Sigma$ equipped with a basepoint, and the following additional structure: each boundary component is linked to the basepoint by a path, and each boundary component $c$ is labelled with a + or - , and a group element $k_{c}$ of $K$. We interpret the sign to mean that the boundary component $c$ has an intrinsic orientation that agrees, or disagrees, with the induced orientation from $\Sigma$; the intrinsic orientation of the boundary component $c$ gives us a preferred generator for $\pi_{1}(c) \subset \pi_{1}(\Sigma)$, which, by slight abuse of notation, we will also denote by $c$. We define

$$
\operatorname{Hom}(\Sigma, K)=\left\{g \in \operatorname{Hom}\left(\pi_{1}(\Sigma), K\right):\left.g\right|_{c}=k_{c}, \forall c \in \pi_{0}(\partial \Sigma)\right\} .
$$

This space only depends upon the basepoint and paths to the boundary components up to homotopy. The pure mapping class group $\Gamma_{\Sigma}$ does not in general act on this space; only the subgroup generated by Dehn twists along curves which do not cross the paths from the basepoint to the boundary components will act; we denote this group by $\pi_{0}(\operatorname{Aut}(\Sigma))$.

If $\Sigma$ is a closed surface, then we can form the quotient of $\operatorname{Hom}(\Sigma, K)$ by the global gauge action of $K$ by conjugation; the quotient is denoted by $H^{1}(\Sigma, K)$. In this case, $\pi_{0}(\operatorname{Aut}(\Sigma))=\Gamma_{\Sigma}$, the mapping class group.

Let $s$ denote a separating oriented simple closed curve on $\Sigma$. We suppose that the basepoint is on $s$, and we suppose also that $s$ does not cut any of the paths from the basepoint to the boundary components. Let $\check{\Sigma}_{k}=\Sigma_{k}^{-} \sqcup \Sigma_{k}^{+}$denote the disconnected object obtained by cutting along $s$ and attaching one - and one + , and same group element $k$, to the new boundary components. The Seifert-Van Kampen Theorem implies that the projection $p: \Sigma \rightarrow \Sigma$ induces an exact sequence

$$
0 \rightarrow\langle s\rangle \rightarrow \pi_{1}\left(\Sigma^{-}\right) * \pi_{1}\left(\Sigma^{+}\right) \stackrel{p_{*}}{\longrightarrow} \pi_{1}(\Sigma) \rightarrow 0 .
$$

where $\langle s\rangle$ denotes the normal subgroup generated by the element $s^{-1} * s$. Hence we have the following elementary

Sewing Lemma (1.3). Assume $\Sigma$ has a group element boundary condition. Then there is a bijective correspondence

$$
\operatorname{Hom}(\Sigma, K)=\bigsqcup_{k \in K} \operatorname{Hom}\left(\Sigma_{k}^{-}, K\right) \times \operatorname{Hom}\left(\Sigma_{k}^{+}, K\right),
$$

where $g \leftrightarrow\left(g^{-}, g^{+}\right), g^{-}(s)=g^{+}(s)=k, g^{ \pm}=\left.g\right|_{\pi_{1}\left(\Sigma^{ \pm}\right)}$. This correspondence is equivariant with respect to $\pi_{0}(\operatorname{Aut}(\check{\Sigma}))$, the group generated by Dehn twists along curves which cross neither $s$ nor the paths from basepoint to boundary components. 


\section{Initial cases}

The basic insight of this paper is that in all cases involving boundary, $\Gamma$-ergodicity is equivalent to $\mathcal{G}$-ergodicity, where $\mathcal{G}$ is a continuous group of volume-preserving transformations, for a.e. boundary condition. The latter problem reduces to a calculation concerning infinitesimal transitivity.

\subsection{The one-holed torus, with group element boundary condition}

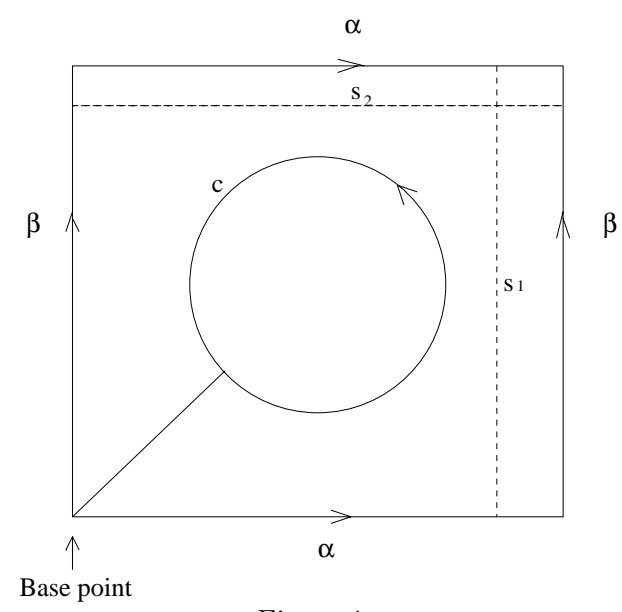

Figure 1

In this subsection we let $\Sigma$ denote the one-holed torus, with boundary component $c$, which we view as in Figure 1.

Given $k \in K$, we write $\Sigma_{k}$ to indicate that we impose the boundary condition $k$, so that $\Sigma_{k}$ is an object of the type considered in Section 1 . We have

$$
\begin{array}{ccc}
\operatorname{Hom}\left(\pi_{1} \Sigma, K\right) \leftrightarrow & K \times K \\
& \downarrow p \\
& K^{\prime}=[K, K]
\end{array} \quad\left(g \leftrightarrow\left(g_{\alpha}, g_{\beta}\right)\right)
$$

where $p$ is the commutator map, $p(g, h)=g h g^{-1} h^{-1}$. With respect to this identification, the fibers of $p$ are precisely the representation spaces $\operatorname{Hom}\left(\Sigma_{k}, K\right)$. Define $\Gamma$ to be the group generated by the transformations $T_{j}: K \times K \rightarrow K \times K$ given by

$$
T_{1}(g, h)=\left(g h^{-1}, h\right), \quad T_{2}(g, h)=\left(g, h g^{-1}\right) .
$$

These transformations arise from twists along the curves $s_{1}$ and $s_{2}$ indicated in Figure 1; they are volume-preserving (with respect to Haar measure), hence naturally induce unitary transformations of $L^{2}(K \times K)$, they commute with conjugation by $K$, and they fix the map $p$. The action of $\Gamma$ restricts to the action of $\pi_{0}\left(\operatorname{Aut}\left(\Sigma_{k}\right)\right)$ on the fiber $p^{-1}(k)=\operatorname{Hom}\left(\Sigma_{k}, K\right)$. 
In this subsection we prove the following result, which is of independent interest.

Theorem (2.1.4). Suppose that $F \in L^{2}(K \times K)$ is $\Gamma$-invariant. Then $F$ is a.e. constant on components of $p^{-1}(k)$ for a.e. $k[d \rho]$, where $d \rho=p_{*}(d g \times d h)$.

We refer to [Bt] for the general theory of representations of compact Lie groups.

Remarks (2.1.5). (a) The measure $d \rho$ is in the Lebesgue class of $K^{\prime}$, and

$$
d \rho(k)=\left(\sum_{\mu} d_{\mu}^{-1} \chi_{\mu}(k)\right) d k
$$

where the sum is over all irreducible characters of $K^{\prime}$, and $d_{\mu}=\chi_{\mu}(1)$. To see this, first note that because $p$ is a conj $(K)$-equivariant map, and $d g \times d h$ is conjugation invariant, $d \rho$ is conjugation invariant. Secondly, if $f=\sum c_{\mu} \chi_{\mu}$ is a central function, then

$$
\begin{aligned}
\int f d \rho & =\int_{K^{\prime}} \int_{K^{\prime}} f\left(g h g^{-1} h^{-1}\right) d g d h=\sum_{\mu} c_{\mu} \int\left\{\int \chi_{\mu}\left(g h g^{-1} h^{-1}\right) d h\right\} d g \\
& =\sum c_{\mu} \int \frac{\left|\chi_{\mu}(g)\right|^{2}}{\chi_{\mu}(1)} d g=\int\left(\sum c_{\nu} \chi_{\nu}(g)\right)\left(\sum d_{\mu}^{-1} \chi_{\mu}\left(g^{-1}\right)\right) d g,
\end{aligned}
$$

which heuristically explains (2.1.6). (The third equality uses the well-known integration formula $\int \chi\left(x h y h^{-1}\right) d h=\chi(x) \chi(y) / \chi(1)$, which follows from observing that the left hand side is a central function for $(x, y) \in K \times K$, and computing the expansion in terms of characters for $K \times K$.) Because characters are orthogonal,

$$
\int\left|\sum d_{\mu}^{-1} \chi_{\mu}(g)\right|^{2} d g=\sum d_{\mu}^{-2} \int\left|\chi_{\mu}(g)\right|^{2} d g=\sum d_{\mu}^{-1} .
$$

The Weyl dimension formula implies that this sum is finite, provided $\mathfrak{k}$ does not have $s u(2)$ factors (see below); hence in most cases, the density in (2.1.6) represents an $L^{2}$ function on $K$. In general, if we fix a maximal torus and positive Weyl chamber, so that we can parameterize the representations by dominant integral functionals $\mu$, then the Weyl character formula implies that for $g \in T$,

$$
\sum d_{\mu}^{-1} \chi_{\mu}(g)=\Delta(g)^{-1} \sum_{w \in W}(-1)^{l(w)}\left\{e^{i \rho} \sum_{\mu} d_{\mu}^{-1} e^{i \mu}\right\}^{w}(g),
$$

where $W$ is the Weyl group, $l(w)$ is the length of $w$, and $\rho$ is half the sum of the positive roots. If we write $\mu$ in terms of the fundamental dominant integral functionals, $\mu=\sum n_{j} \mu_{j}$, then the Weyl dimension formula implies

$$
d_{\mu}=\prod_{\alpha>0} \frac{\langle\mu+\rho, \alpha\rangle}{\langle\rho, \alpha\rangle} \sim \prod_{j} n_{j}^{\left\langle\mu_{j}, 2 \rho\right\rangle},
$$


so that $\sum d_{\mu}^{-1} e^{i \mu}=\sum d_{\mu}^{-1} e^{i \sum n_{j} \theta_{j}}$ always represents an $L^{2}$ function with respect to the Haar measure of $T$. Since this function is the boundary values of a holomorphic function on $\left(\mathbb{C}_{\leq 1}\right)^{r}$, it cannot vanish on a set of positive measure. This explains the meaning of the density (2.1.6), and shows that $d \rho$ is in the Lebesgue class (see Appendix B for a more direct proof).

In the case of $K=S U(2)$, the density in (2.1.6), as a function of $\operatorname{diag}\left(z, z^{-1}\right)$, $z=e^{i \theta}$, is given by

$$
\begin{gathered}
\frac{1}{z-z^{-1}}\left\{z \sum_{d \geq 1} d^{-1} z-z^{-1} \sum_{d \geq 1} d^{-1} z^{-d}\right\}=-\operatorname{Im}\left\{\frac{e^{i \theta} \ln \left(1-e^{i \theta}\right)}{\sin \theta}\right\} \\
=\ln (2-2 \cos \theta)^{1 / 2}+\cos \theta \frac{\arg (1-\cos \theta-i \sin \theta)}{\sin \theta} .
\end{gathered}
$$

(b) Theorem (2.1.4) gives an algebraic characterization of functions $F(g, h)$ which have the form $f\left(g h g^{-1} h^{-1}\right)$, in situations where all the fibers $p^{-1}(k)$ are connected, e.g. for $K$ simply connected (see Appendix A). It seems to be unknown whether there might be a reasonable characterization for more general groups (e.g. finite groups).

Corollary (2.1.7) of Theorem (2.1.4). For a.e. boundary condition $k \in K$, the action

$$
\pi_{0}\left(\operatorname{Aut}\left(\Sigma_{k}\right)\right) \times \operatorname{Hom}\left(\Sigma_{k}, K\right)
$$

is ergodic on the Lebesgue class of each component.

To prove (2.1.4), we need to be able to analyze the transformations in (2.1.3). Let $T=T_{2}$ denote the unitary transformation on $L^{2}\left(K_{1} \times K_{2}\right)$ corresponding to the second of these transformations, where we have introduced copies $K_{1}$ and $K_{2}$ of $K$, for notational clarity. Recall that the Peter-Weyl Theorem asserts that there is a $K \times K$-equivariant isomorphism

$$
\bigoplus_{\mu} \mathcal{L}\left(V_{\mu}\right) \rightarrow L^{2}(K):\left(L_{\mu}\right) \rightarrow f, \quad f(g)=\sum_{\mu} \operatorname{dimn}(\mu)^{1 / 2} \operatorname{tr}_{\mu}\left(L_{\mu} \pi_{\mu}\left(g^{-1}\right)\right),
$$

where $\mathcal{L}\left(V_{\mu}\right)$ denotes the space of linear transformations of $V_{\mu}$, the sums are over all irreducible representations, and the linear action of $\left(g_{l}, g_{r}\right) \in K \times K$ on these respective spaces is given by

$$
\begin{aligned}
L_{\mu} & \rightarrow \pi_{\mu}\left(g_{l}\right) L_{\mu} \pi_{\mu}\left(g_{r}\right)^{-1} \\
f(g) & \rightarrow f\left(g_{l}^{-1} g g_{r}\right) .
\end{aligned}
$$

Lemma (2.1.9). Via the isomorphisms

$$
L^{2}\left(K_{1} \times K_{2}\right)=L^{2}\left(K_{1} ; L^{2}\left(K_{2}\right)\right)=\bigoplus_{\mu} L^{2}\left(K_{1} ; \mathcal{L}\left(V_{\mu}\right)\right)
$$


$T=\operatorname{diag}\left(T_{\mu}\right)$, where $T_{\mu}$ is the multiplication operator

$$
T_{\mu}: L^{2}\left(K_{1} ; \mathcal{L}\left(V_{\mu}\right)\right) \rightarrow L^{2}\left(K_{1} ; \mathcal{L}\left(V_{\mu}\right)\right): F_{\mu}(g) \rightarrow F_{\mu}(g) \pi_{\mu}(g)^{-1} .
$$

In particular

$$
L^{2}\left(K_{1} ; \mathcal{L}\left(V_{\mu}\right)\right)^{T_{\mu}}=\left\{F_{\mu}:\left.F_{\mu}(g)\right|_{\left(V_{\mu}^{g}\right)^{\perp}}=0, \text { a.e. } g\right\},
$$

and if $F_{\mu}$ is $T_{\mu}$-invariant, then (viewed now as a function of two variables)

$$
F_{\mu}(g, h)=F_{\mu}\left(g, h a(g)^{-1}\right),
$$

for any measurable function $a: K \rightarrow K$ such that $[a(g), g]=1$ a.e..

Proof of Lemma (2.1.9). The formula for $T_{\mu}$ is a direct consequence of the PeterWeyl theorem, and the other statements follow directly from the formula for $T_{\mu}$.

Note that

$$
A=\{a: K \rightarrow K:[g, a(g)]=1, \forall g\}
$$

is an abelian subgroup of the gauge group $\operatorname{Map}(K, K)$. We will assume that the maps in $A$ are smooth, unless noted otherwise. It is probably not the case that $A$ is a Lie subgroup, because the family of projections onto the subalgebras $\mathfrak{k}^{g}$, $g \in K$, is not smooth. Nonetheless we will refer to

$$
\mathfrak{a}=\left\{x: K \rightarrow \mathfrak{k}: \operatorname{Ad}_{g}(x(g))=x(g), \forall g\right\}
$$

as the Lie algebra of $A$, because it has the crucial property

$$
\exp (\mathfrak{a}) \subset A
$$

The group $A$ acts on $K_{1} \times K_{2}$ in two ways, corresponding to the actions (2.1.3), by

$$
A_{1}(a):(g, h) \rightarrow\left(g a(h)^{-1}, h\right), \quad A_{2}(a):(g, h) \rightarrow\left(g, h a(g)^{-1}\right),
$$

respectively. Note that the transformations $T_{i}^{n}, i=1,2$, correspond to $a(k)=k^{n}$. Note also that the transformations (2.1.15) are volume-preserving.

We can restate $(2.1 .9)$ as

Lemma (2.1.16). The $L^{2}$ function $F(g, h)$ is $T_{j}$-invariant if and only if $F$ is $A_{j}$-invariant, for $j=1,2$. (Here we can require the maps in $A$ to be $C^{\infty}, C^{0}$, or merely measurable - the basic result is insensitive to this requirement.) 
Let $\mathcal{G}$ denote the closure of the group of volume-preserving transformations of $K \times K$ generated by $A_{1}$ and $A_{2}$, inside the Lie group of all volume-preserving diffeomorphisms of $K \times K$ (it will turn out that, for our purposes, we could just as well consider the closure in the group of all volume-preserving transformations, in the natural strong operator topology). It is unclear whether $\mathcal{G}$ is a Lie group, but it is useful to think in these terms, as we will now see. The Lie algebra actions corresponding to $(2.1 .15)$ are given by the vector fields on $K_{1} \times K_{2}$

$$
\left.d A_{1}(x)\right|_{g, h}=(-x(h), 0),\left.\quad d A_{2}(x)\right|_{g, h}=(0,-x(g)),
$$

respectively, for $x \in \mathfrak{a}$. These actions do not necessarily commute.

Definitions (2.1.18). (a) $\mathfrak{g}_{0}$ is the Lie algebra of vector fields on $K \times K$ given by

$$
\mathfrak{g}_{0}=\left\{\left(\left.x\right|_{h},\left.y\right|_{g}\right): x, y \in \mathfrak{a}\right\}
$$

(b) $\mathfrak{g}$ is the Lie algebra of vector fields on $K \times K$ generated by the family of Lie algebras

$$
\left\{\operatorname{Ad}_{\sigma} \mathfrak{g}_{0}: \sigma \in A_{1} \quad \text { or } \quad A_{2}\right\}
$$

The bracket for $\mathfrak{g}_{0}$ is given by

$$
\left.\left[\left(x_{1}, y_{1}\right),\left(x_{2}, y_{2}\right)\right]\right|_{g, h}=\left(\left.d x_{2}\left(y_{1}\right)\right|_{h}-\left.d x_{1}\left(y_{2}\right)\right|_{h},\left.d y_{2}\left(x_{1}\right)\right|_{g}-\left.d y_{1}\left(x_{2}\right)\right|_{g}\right)
$$

(see (0.2)).

Heuristically $\mathfrak{g}_{0}$ is the Lie algebra corresponding to the group generated by the identity components of $A_{1}$ and $A_{2}$, while heuristically $\mathfrak{g}$ is the Lie algebra corresponding to $\mathcal{G}$. In practice we will think of $\mathfrak{g}$ as an $\operatorname{Ad}(\Gamma)$-invariant Lie algebra containing $\mathfrak{g}_{0}$.

Lemma (2.1.20). Assuming we require maps to be $C^{\infty}$, we have $\exp (\mathfrak{g}) \subset \mathcal{G}$.

Proof of Lemma (2.1.20). Suppose that $\xi=(y, x) \in \mathfrak{g}_{0}$. Now $\exp \{t(0, x)\} \in A_{2}$ and $\exp \{t(y, 0)\} \in A_{1}, \forall t$. Thus

$$
\exp (\xi)=\lim _{n \rightarrow \infty}\left(\exp ((y / n, 0) \exp (0, x / n))^{n} \in \mathcal{G},\right.
$$

because Trotter's product formula (see $(2.12 .5)$ of $[\mathrm{V}]$ ) is valid for vector fields on a compact manifold. Therefore for any $\sigma \in A_{j}$,

$$
\exp \left(\operatorname{Ad}_{\sigma}(\xi)\right)=\sigma \exp (\xi) \sigma^{-1} \in \mathcal{G}
$$

Using Trotter's product formula (and the analogue for brackets) in the same way, we see that for sums and brackets of such vector fields, we again exponentiate into $\mathcal{G}$. 
Our goal now is to show that the Lie algebra $\mathfrak{g}$ is infinitesimally transitive off a set of codimension $>1$ along a generic fiber of the commutator map $p$. We calculate that

$$
\begin{aligned}
\left.d p\right|_{g, h}: \mathfrak{k} \oplus \mathfrak{k} & \rightarrow \mathfrak{k}^{\prime}:(\xi, \eta) \rightarrow \xi^{h g h^{-1}}-\xi^{h g}+\eta^{h g}-\eta^{h} \\
& =\left(\xi^{h^{-1}}-\xi+\eta-\eta^{g^{-1}}\right)^{h g}
\end{aligned}
$$

Proposition (2.1.24). For $g$ and $h$ in the complement of a set of codimension $>1$, the evaluation map

$$
\text { eval }\left.\right|_{g, h}: \mathfrak{g} \rightarrow \operatorname{ker}\left(\left.d p\right|_{(g, h)}\right)
$$

is surjective, where eval is the evaluation map.

Proof of Proposition (2.1.24). If $K$ is abelian, then at all points

$$
\text { eval }\left.\right|_{g}: \mathfrak{a} \rightarrow \mathfrak{k}^{\operatorname{Ad}(g)}
$$

is surjective, and it follows from this that $\mathfrak{g}$ is transitive.

So suppose that $K$ is nonabelian. Recall the set of regular points,

$$
K^{\text {reg }}=\left\{k \in K: \operatorname{dimn}\left(\mathfrak{k}^{g}\right)=r\right\}
$$

where $r=\operatorname{rank}(\mathfrak{k})$ is the minimal possible dimension of $\mathfrak{k}^{g}$. The singular set $K \backslash K^{\text {reg }}$ has codimension 3 , because for a nonregular point $g, \mathfrak{k}^{g}$ always contains a copy of $s u(2)$, in addition to a maximal torus. For a regular point $g \in K$, eval $\left.\right|_{g}$ in (2.1.25) will be surjective (while the image shrinks at nonregular points, e.g. eval $\left.\left.\right|_{1}(\mathfrak{a})=\{0\}\right)$; this follows from the real analyticity of the vector bundle $g \rightarrow \mathfrak{k}^{g}$ over $K^{\text {reg }}$. Therefore for $g, h \in K^{\mathrm{reg}}$,

$$
\text { eval }\left.\right|_{g, h}\left(\mathfrak{g}_{0}\right)=\mathfrak{k}^{h} \oplus \mathfrak{k}^{g} \subset \operatorname{ker}\left(\left.d p\right|_{g, h}\right) \subset \mathfrak{k} \oplus \mathfrak{k}
$$

This always fills out the central part of $\mathfrak{k}$. For this reason, without loss of generality, we can henceforth assume that $\mathfrak{k}$ is semisimple.

The map $p$ is regular at all points $(g, h)$ such that $\mathfrak{k}^{g} \cap \mathfrak{k}^{h}=\{0\}$, by (2.1.23). The abstract meaning of this condition is that the representation of $\pi_{1}(\Sigma)$ determined by $(g, h)$ is irreducible, in the intrinsic sense that the commutant of the image in $K$ is the center of $K$. To understand this condition more concretely (from a point of view useful to us), suppose that $g$ and $h$ are regular. Write $h=\exp (y)$, so that $\mathfrak{k}^{h}=C_{\mathfrak{k}}(y)$, the centralizer of $y$. For $x \in \mathfrak{k}^{g}$,

$$
[x, y]=\sum_{\alpha} \alpha(x) y_{\alpha}
$$


where the sum is over all roots $\alpha$ of $\mathfrak{k}^{g}$, and $y_{\alpha}$ denotes the component of $y$ in the root space of $\alpha$. In order for $x \in \mathfrak{k}^{h}$, we must have $\alpha(x)=0$, whenever $y_{\alpha} \neq 0$. The condition $y_{\alpha}=0$ is two independent real conditions, because the root space has one complex dimension. Thus $\left\{(g, h): \mathfrak{k}^{g} \cap \mathfrak{k}^{h} \neq\{0\}\right\}$ has codimension at least 2 .

We now know that the dimension of $\operatorname{ker}(d p)$ is dimn $(\mathfrak{k})$ off a subset of codimension 2. Let $\operatorname{proj}_{i}$ denote projection onto the $i$ th factor. The map proji induces an exact sequence

$$
0 \rightarrow\left\{\left(0, \mathfrak{k}^{g}\right)\right\} \rightarrow \operatorname{ker}(d p) \stackrel{\operatorname{proj}_{1}}{\longrightarrow}\left\{\xi \in \mathfrak{k}:\left(1-\operatorname{Ad}\left(h^{-1}\right)\right) \xi \in\left(\mathfrak{k}^{g}\right)^{\perp}\right\} \rightarrow 0 ;
$$

there is a similar sequence for $\operatorname{proj}_{2}$. The evaluation of $\mathfrak{g}_{0}$ at $(g, h) \in K^{\mathrm{reg}} \times K^{\mathrm{reg}}$ fills out $\operatorname{ker}\left(\operatorname{proj}_{1}\right)+\operatorname{ker}\left(\operatorname{proj}_{2}\right)$. Since

$$
\left\{\xi \in \mathfrak{k}:\left(1-\operatorname{Ad}\left(h^{-1}\right)\right) \xi \in\left(\mathfrak{k}^{g}\right)^{\perp}\right\}^{\perp}=(1-\operatorname{Ad}(h)) \mathfrak{k}^{g},
$$

to prove that eval $: \mathfrak{g} \rightarrow \operatorname{ker}(d p)$ is surjective at a regular point, it suffices to prove that

$$
(1-\operatorname{Ad}(h)) \mathfrak{k}^{g}+\operatorname{proj}_{1}\left(\left.\operatorname{eval}\right|_{g, h}(\mathfrak{g})\right)=\mathfrak{k} ;
$$

there is a similar statement for $\operatorname{proj}_{2}$.

Now $\mathfrak{g}$ is $\Gamma$-invariant, hence

$$
\left.\sum_{\Gamma} \gamma_{*}\left(\text { eval }\left.\right|_{\gamma^{-1}(g, h)}\left(\mathfrak{g}_{0}\right)\right) \subset \operatorname{eval}\right|_{g, h}(\mathfrak{g})
$$

In geometric terms, the sum is the $\Gamma$-invariant distribution generated by $\mathfrak{g}_{0}$. We will first consider only a small part of this sum, namely the $T_{2}$-invariant distribution generated by $\mathfrak{g}_{0}$.

Suppose that $\left(\left.y\right|_{h},\left.x\right|_{g}\right) \in \mathfrak{g}_{0}$. We have

$$
\begin{aligned}
& \left(T_{2}^{n}\right)_{*}\left(\text { eval }\left.\right|_{T_{2}^{-n}(g, h)}(y, x)\right) \\
& =\left(0,\left.x\right|_{g}\right)+\left.\frac{d}{d t}\right|_{t=0}\left(g e^{t y\left(h g^{n}\right)}, h g^{n}\left(g e^{t y\left(h g^{n}\right)}\right)^{-n}\right) \\
& = \begin{cases}\left(y\left(h g^{n}\right), x(g)-\sum_{k=1}^{n} y\left(h g^{n}\right)^{g^{k}}\right), & n>0 \\
\left(y\left(h g^{n}\right), x(g)+\sum_{k=n+1}^{0} y\left(h g^{n}\right)^{g^{k}}\right), & n<0 .\end{cases}
\end{aligned}
$$

From this we see that

$$
\sum_{h g^{n} \in K^{\text {reg }}} \mathfrak{k}^{h g^{n}} \subset \operatorname{proj}_{1}\left(\left.\operatorname{eval}\right|_{g, h}(\mathfrak{g})\right) .
$$

Now for $(g, h) \in K^{\mathrm{reg}} \times K^{\mathrm{reg}}$, if $\left\{g^{n}\right\}$ is a dense subgroup of $T=\exp (\mathfrak{t})$, then

$$
\sum_{\left\{n: h g^{n} \in K^{\mathrm{reg}}\right\}} \mathfrak{k}^{h g^{n}}=\sum_{\left\{x \in \mathfrak{t}: h e^{x} \in K^{\mathrm{reg}}\right\}} \mathfrak{k}^{h e^{x}} .
$$


For we clearly have $\subset$. Conversely given $x \in \mathfrak{t}$ such that $h e^{x}$ is regular, we can find a sequence $\left\{n_{j}\right\}$ such that $g^{n_{j}} \rightarrow e^{x}$ as $j \rightarrow \infty$, hence $h g^{n_{j}}$ will be regular for $j$ sufficiently large, and $\mathfrak{k}^{h g^{n_{j}}} \rightarrow \mathfrak{k}^{h e^{x}}$, so that the opposite inclusion holds.

Lemma (2.1.34). There is a set $X_{2} \subset K$ of real codimension $\geq 2$ such that for $(g, h) \notin K \times X_{2}$,

$$
(1-\operatorname{Ad}(h)) \mathfrak{k}^{g}+\sum_{\left\{x \in \mathfrak{k}^{g}: h e^{x} \in K^{\mathrm{reg}}\right\}} \mathfrak{k}^{h e^{x}}=\mathfrak{k} .
$$

Proof of Lemma (2.1.34). We write $h=\exp (y)$. We also write $\mathfrak{t}=\mathfrak{k}^{g}$. Since $h$ is regular, there are open neighborhoods $\mathfrak{u}$ and $U$ of $y$ and $h$, respectively, such that $\exp : \mathfrak{u} \rightarrow U$ is an isomorphism; let log denote the inverse. There is a Taylor series expansion of the form

$$
\log \left(h e^{x}\right)=\sum_{n \geq 0} c_{n}(h, x),
$$

where $c_{n}$ is homogeneous of degree $n$ in $x$. If $|\operatorname{ad}(y)|<\pi$, where $|\cdot|$ denotes the operator norm, then we can also expand each $c_{n}$, and the form of these expansions can be read off from the Baker-Campbell-Hausdorff formula, namely $c_{0}=y$,

$$
c_{1}=x+\frac{1}{2}[y, x]+\frac{1}{12}[y,[y, x]]+\cdots=x+O(|y|),
$$

and for $n>1$,

$$
c_{n}=\text { constant } * \operatorname{ad}(x)^{n}(y)+o(|y|),
$$

as $|y| \downarrow 0$, where the constant depends only upon $n$. We also have

$$
(1-\operatorname{Ad}(h)) x=[x, y]+o(|y|) \quad \text { as } \quad|y| \downarrow 0 .
$$

We now claim that the sum in (2.1.35) equals

$$
\begin{aligned}
& =(1-\operatorname{Ad}(h)) \mathfrak{t}+\mathfrak{k}^{h}+\operatorname{span}\left\{\log \left(h e^{x}\right): x \in \mathfrak{t}, h e^{x} \in U \cap K^{\text {reg }}\right\} \\
& =(1-\operatorname{Ad}(h)) \mathfrak{t}+\mathfrak{k}^{h}+\operatorname{span}\left\{c_{n}(h, x): n \geq 0, x \in \mathfrak{t}\right\} .
\end{aligned}
$$

The first equality is immediate; the second follows from the fact that the span of the power series (2.1.36) will contain the span of the coefficients (replace $x$ by $s x$, note that $h e^{s x} \in U \cap K^{\text {reg }}$ for small $s$, and differentiate with respect to $s$ at $s=0$ ).

Now we first show that (2.1.35) holds for $h \in K^{\text {reg }}$ where $|y|$ is small. As in $(2.1 .28), \mathfrak{k}^{h}=C_{\mathfrak{k}}(y)$, and we can write

$$
y=y_{\mathfrak{t}}+\sum_{\alpha} y_{\alpha}
$$


relative to the root decomposition of $\mathfrak{k}^{\mathbb{C}}$ with respect to $\mathfrak{t}$. If all the $y_{\alpha} \neq 0$, then together $\mathfrak{t}$ and $\left\{\operatorname{ad}(x)^{n}(y): x \in \mathfrak{t}, n \geq 1\right\}$ will span $\mathfrak{k}$. But (2.1.37), (2.1.38) and (2.1.39) now imply that $\left\{c_{n}(h, x): n \geq 1, x \in \mathfrak{t}\right\}$ and $(1-\operatorname{Ad}(h)) \mathfrak{t}$ will span $\mathfrak{k}$, provided that $|y|$ is small.

Note that the condition $y_{\alpha}=0$ is linear, and describes a subset of codimension 2 , as we pointed out below (2.1.28). Thus for $h$ not in a subset of codimension 2 in a neighborhood of 1 , the equation in (2.1.35) holds. It remains to do a similar analysis for a neighborhood of a point $e^{y_{0}} \neq 1$.

Suppose that $h=e^{y_{0}+z}$, where $\log \left(e^{y_{0}}\right)=y_{0}$ and $z$ is small. We have $c_{0}(h, x)=$ $y_{0}+z$,

$$
\begin{gathered}
c_{1}(h, x)=c_{1}\left(e^{y_{0}}, x\right)+\frac{1}{2}[z, x]+o(|z|)=c_{1}\left(e^{y_{0}}, x\right)+O(|z|) \\
(1-\operatorname{Ad}(h)) x=\left(1-\operatorname{Ad}\left(e^{y_{0}}\right)\right) x+[x, z]+o(|z|) \\
c_{n}(h, x)=c_{n}\left(e^{y_{0}}, x\right)+\text { constant } * \operatorname{ad}(x)^{n}(z)+o(|z|)
\end{gathered}
$$

as $|z| \downarrow 0$, where $n>1$. The derivative of $\mathfrak{k}^{h}$ (as it varies in the Grassmannian of subspaces, $\operatorname{Gr}(r, \mathfrak{k}))$ is a linear transformation $T(z): C\left(y_{0}\right) \rightarrow C\left(y_{0}\right)^{\perp}$. If $T(z)\left(\xi_{0}\right)=\xi_{1}$, then to first order in $s, \exp \left(\operatorname{ad}\left(y_{0}+s z\right)\right)\left(\xi_{0}+s \xi_{1}\right)=\xi_{0}+s \xi_{1}$, i.e. $\left[y_{0}, \xi_{1}\right]+\left[z, \xi_{0}\right]=0$; in terms of the root decomposition for $C\left(y_{0}\right)$, we have

$$
T(z)(\xi)=\sum_{\beta} \frac{\beta(\xi)}{\beta\left(y_{0}\right)} z_{\beta} .
$$

Thus

$$
\mathfrak{k}^{h}=\operatorname{graph}\left(T(z): C\left(y_{0}\right) \rightarrow C\left(y_{0}\right)^{\perp}\right)+o(|z|)
$$

as $|z| \downarrow 0$.

Now consider the possibility that together $\mathfrak{k}^{\exp \left(y_{0}\right)},\left(1-\operatorname{Ad}\left(e^{y_{0}}\right)\right) \mathfrak{t}$, and $\left\{c_{n}\left(e^{y_{0}}, x\right)\right.$ : $n \geq 0, x \in \mathfrak{t}\}$ do not span $\mathfrak{k}$. The argument proceeds initially as in the case $\exp \left(y_{0}\right)=1$. If $\forall \alpha, z_{\alpha} \neq 0$ (the components with respect to the root decomposition for $\mathfrak{t})$, then $\left\{\operatorname{ad}(x)^{n}(z): n \geq 1, x \in \mathfrak{t}\right\}$ will span $\mathfrak{t}^{\perp}$. We now use (2.1.42)-(2.1.44). For the variation of the span of $\mathfrak{k}^{h},(1-\operatorname{Ad}(h)) \mathfrak{t}$, and $\left\{c_{n}(h, x): n \geq 0, x \in \mathfrak{t}\right\}$ to be all of $\mathfrak{k}$, it is therefore sufficient for the natural map of an $r+1$-dimensional space to an $r$-dimensional space

$$
\mathbb{R} z+\operatorname{graph}(T(z)) \rightarrow \mathfrak{k} / \mathfrak{t}^{\perp}
$$

to be surjective (note that the $z$ comes from the $c_{0}$ term; see the line preceding (2.1.42)). Thus if (1) $z_{\alpha} \neq 0, \forall \alpha$, and (2) (2.1.47) is surjective, then for $h$ corresponding to small $z,(2.1 .35)$ will hold.

We have already remarked that the first condition describes a subset of codimension 2. From the formula (2.1.45) for $T(z)$, we see that $z$ is generically independent of graph $(T(z))$, and graph $(T(z))$ is generically transverse to $\mathfrak{t}^{\perp}$. Therefore 
the condition (2) also describes a subset of codimension 2, for $z$ in a small neighborhood of 1 . This completes the proof.

We can now continue with the proof of Proposition (2.1.24). Let $Q$ denote the set of points $g$ in $K^{\text {reg }}$ with the property that $\left\{g^{n}\right\}$ is not dense in the torus $\exp \left(\mathfrak{k}^{g}\right)$.

Now by $(2.1 .33)$ and $(2.1 .34)$ we know that eval $\left.\right|_{(g, h)}$ maps onto $\operatorname{ker}(d p)$ provided that $(g, h)$ is not in the set $\left(Q_{1} \times K_{2}\right) \cup\left(K_{1} \times X_{2}\right)$. Now by considering the $T_{1}$-invariant distribution generated by $\mathfrak{g}_{0}$ (and proj2), we can also conclude that eval $\left.\right|_{(g, h)}$ maps onto $\operatorname{ker}(d p)$ provided that $(g, h)$ is not in the set $\left(K_{1} \times Q_{2}\right) \cup\left(X_{1} \times K_{2}\right)$.

Because the condition that eval $\left.\right|_{(g, h)}$ maps onto $\operatorname{ker}(d p)$ is a linear independence condition, involving real analytic vector fields, the set of points where eval does not map onto $\operatorname{ker}(d p)$ is generically real analytic, and by the proceding paragraph of codimension $\geq 1$. Since $X_{1}$ and $X_{2}$ have codimension $\geq 2$, the only portion of the singular set identified in the previous paragraph which could a priori support an object of codimension one is $Q_{1} \times Q_{2}$. But $Q$ has Hausdorff dimension $d-1$, where $d$ is the dimension of $K$, hence $Q_{1} \times Q_{2}$ has Hausdorff dimension $2 d-2$. So the singular set must have codimension at least 2. This completes the proof of (2.1.24).

Proof of Theorem (2.1.4). Suppose that $F \in L^{2}(K \times K)$ is $\Gamma$-invariant. By (2.1.16) $F$ is $\mathcal{G}$-invariant. Now given a generic point where $\mathfrak{g}$ is infinitesimally transitive along the fiber, the $\mathcal{G}$-orbit of that point will be open in the fiber. For a generic fiber, the complement of these open sets has codimension $>1$, by (2.1.24). Hence for a generic fiber, the $\mathcal{G}$-orbits necessarily coincide with the components of the fiber. Thus an invariant $F$ is locally constant on connected components of a.e. fiber.

\subsection{The $n$-holed torus, with group element boundary condition}

$\alpha$

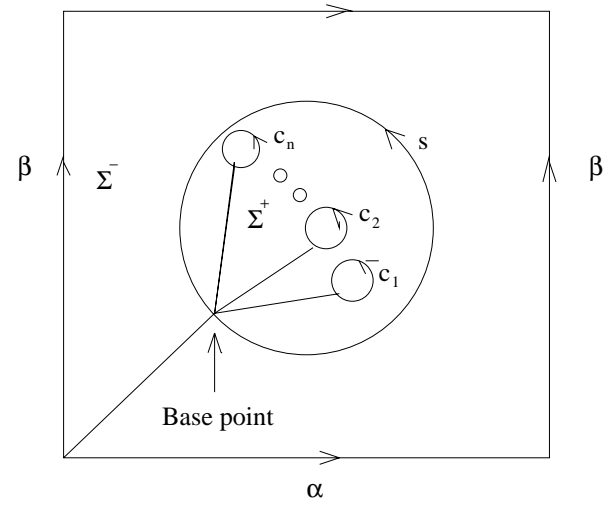

Figure 2 
Let $\Sigma_{\left(k_{1}, \ldots, k_{n}\right)}$ denote the $n$-holed torus with boundary components $c_{1}, \ldots, c_{n}$, as in Figure 2 (where we ignore $s$ momentarily), with group element boundary condition $k_{c_{j}}=k_{j}, 1 \leq j \leq n$. Let $\Sigma_{k}^{-}$denote the one-holed torus with group element boundary condition considered in Section 2.1, which reappears in Figure 2 with boundary component $s$ (and where we have moved the basepoint from the vertex to $s$, which we can do without affecting the results of Section 2.1). Let $\Sigma_{\left(k^{\prime}, k_{1}, \ldots, k_{n}\right)}^{+}$denote the $n+1$-holed sphere with group element boundary condition pictured in Figure 2, where $k^{\prime}$ is the labeling for $s$, and $k_{j}$ is the label for $c_{j}$; the corresponding Hom space is empty unless $k^{\prime}=\prod k_{j}$, in which case it is a point. The Sewing Lemma (1.3) implies that we have a $\pi_{0}\left(\operatorname{Aut}\left(\Sigma^{-}\right)\right)$-equivariant bijection

$$
\operatorname{Hom}\left(\Sigma_{\left(k_{1}, \ldots, k_{n}\right)}, K\right) \leftrightarrow \operatorname{Hom}\left(\Sigma_{k}^{-}, K\right) \times \operatorname{Hom}\left(\Sigma_{\left(k, k_{1}, \ldots, k_{n}\right)}^{+}, K\right)
$$

where $k=\prod k_{j}$. Note that $\pi_{0}\left(\operatorname{Aut}\left(\Sigma^{-}\right)\right)=\pi_{0}(\operatorname{Aut}(\Sigma))$.

Unfortunately this is not a situation where we can integrate over $k$, to obtain a result for every boundary condition, because $k$ is fixed by the $k_{j}$. We need to vary one of the boundary conditions, say $k_{n}$. We write $\Sigma_{(\vec{k}, \cdot)}$ for the object with boundary $k_{c_{j}}=k_{j}, 1 \leq j<n$, where we allow $k_{c_{n}}$ to vary. We then have a $\pi_{0}(\operatorname{Aut}(\Sigma))$-equivariant bijection

$$
\operatorname{Hom}\left(\pi_{1} \Sigma^{-}, K\right) \leftrightarrow \operatorname{Hom}\left(\Sigma_{(\vec{k}, \cdot)}, K\right)
$$

An immediate consequence of Theorem (2.1.4) is the following

Corollary (2.2.3). For a.e. $k_{n}[d \rho]$, the action

$$
\pi_{0}(\operatorname{Aut}(\Sigma)) \times \operatorname{Hom}\left(\Sigma_{\left(\vec{k}, k_{n}\right)}, K\right)
$$

is ergodic on the Lebesgue class of each connected component. 


\section{Proof of ergodicity}

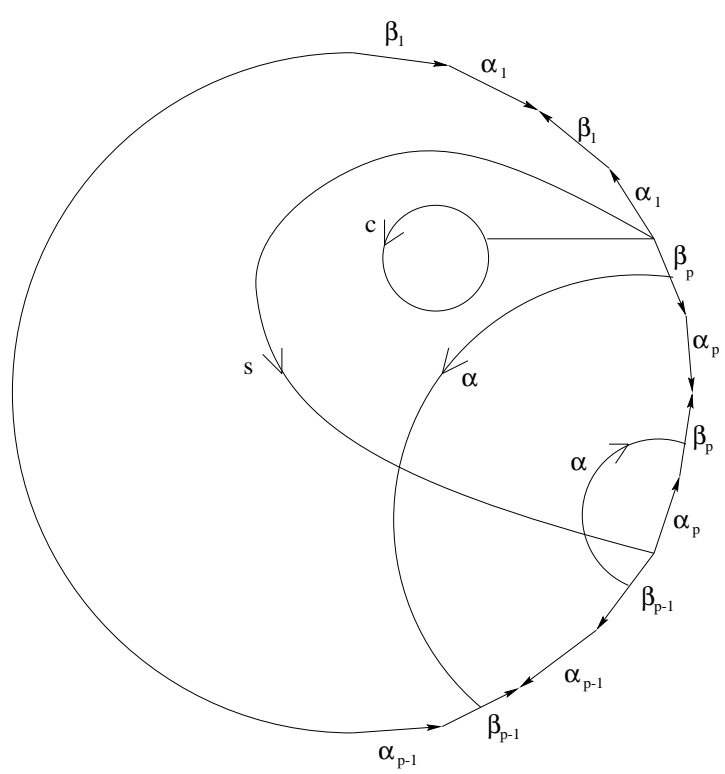

Figure 3

Let $\Sigma$ denote the one-holed surface of genus $p$ with basepoint and link to the boundary $c$, as depicted in Figure 3 (ignore the paths $s$ and $\alpha$ at this point).

Theorem (3.1). If the genus $p>1$, then for every group element boundary condition $k \in K^{\prime}$, the action

$$
\pi_{0}\left(\operatorname{Aut}\left(\Sigma_{k}\right)\right) \times \operatorname{Hom}\left(\Sigma_{k}, K\right) \rightarrow \operatorname{Hom}\left(\Sigma_{k}, K\right)
$$

is ergodic with respect to the Lebesgue class of each connected component.

Note that Theorem (0.1) (when the genus $>1$ ) is the special case $k=1$ of (3.1). When the surface in (0.1) has genus $=1$, then (0.1) essentially reduces to the abelian case, and this is a standard application of Fourier series.

The basic facts about the connectedness properties of $\operatorname{Hom}(\Sigma, K)$ which we will require are gathered in Appendix $\mathrm{A}$, for the convenience of the reader. In particular (A.3) asserts that $\pi_{0}(\operatorname{Aut}(\Sigma))$ acts on components, so that the statement of the Theorem makes sense.

Proof of Theorem (3.1). Consider the decomposition of $\operatorname{Hom}(\Sigma, K)$ into connected components described in (A.3). If we prove (3.1) for all groups of the form $T \times K_{1}$, where $T$ is a torus and $K_{1}$ is simply connected, then we will be done. So henceforth 
we assume that $K^{\prime}$ is simply connected. In this case all the representation spaces are connected, by (A.2).

We have proven that $\pi_{0}\left(\operatorname{Aut}\left(\Sigma^{-}\right)\right)$acts ergodically on $\operatorname{Hom}\left(\Sigma^{-}, K\right)$, for a oneholed torus $\Sigma^{-}$as in Section 2.1, for a.e. group element boundary condition. Similarly we have proven that $\pi_{0}\left(\operatorname{Aut}\left(\Sigma^{+}\right)\right)$acts ergodically on $\operatorname{Hom}\left(\Sigma^{+}, K\right)$ for a two-holed torus $\Sigma^{+}$as in Section 2.2 , for a.e. boundary condition on one end, and for every boundary condition on the other end. It therefore suffices to prove the following: suppose that $s$ is a separating curve as in the Sewing Lemma (1.3), such that $\Sigma^{+}$is a two-holed torus; if $\pi_{0}(\operatorname{Aut}(\check{\Sigma}))$ acts ergodically on components of $\operatorname{Hom}(\check{\Sigma}, K)$, for a.e. boundary condition on $s$, then the conclusion of (3.1) holds (see Figure $3 ; \Sigma^{+}$is to the reader's right of $s$ ).

Let $k_{c}$ denote the fixed boundary condition for $\Sigma$. The measure classes for possible boundary conditions on $s$ are the same for $\Sigma^{ \pm}$, the Lebesgue class on $K^{\prime}$ (see $\left.(2.1 .5)\right)$. Let $F$ denote a characteristic function on $\operatorname{Hom}(\Sigma, K)$. If $F$ is

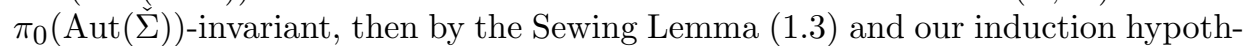
esis, it follows that $F$ is constant along a.e. fiber; hence $F$ is of the form $f\left(\left.g\right|_{s}\right)$, where $f$ is a characteristic function on $K^{\prime}$.

Now suppose that $F$ is $\pi_{0}(\operatorname{Aut}(\Sigma))$-invariant. As in Figure 3, we choose a Dehn twist $\sigma$ corresponding to a loop $\alpha$ that will cross the curve $s$, but will not cross the link to the boundary component, so that $\sigma \in \pi_{0}(\operatorname{Aut}(\Sigma))$. Now the loop $\alpha$ does not pass through the basepoint. There are two elementary ways in which we can use $s$ to link $\alpha$ to the basepoint; if we go from the basepoint in the negative direction along $s$ to $\alpha$, around $\alpha$, and return to the basepoint, then we denote this based loop by $\bar{\alpha}$; if we go from the basepoint in the positive direction along $s$ to $\alpha$, around $\alpha$, and return, then we denote the loop by $\underline{\alpha}$. Using Figure 3 we compute that

$$
\begin{gathered}
\bar{\alpha}=\alpha_{p}\left[\beta_{p}, \alpha_{p}\right] \beta_{p-1} \alpha_{p-1} \beta_{p-1}^{-1}, \quad \underline{\alpha}=s \beta_{p-1} \alpha_{p-1} \beta_{p-1}^{-1} \alpha_{p}\left[\alpha_{p}, \beta_{p}\right]^{-1} s^{-1} \\
\sigma \circ \alpha_{j}=\alpha_{j}, \quad \sigma \circ \beta_{p-1}=\bar{\alpha} \beta_{p-1}, \quad \sigma \circ \beta_{p}=\beta_{p} \bar{\alpha}^{-1} \\
\sigma \circ s=\underline{\alpha} s \bar{\alpha}^{-1}=\left(\prod_{1}^{p-2}\left[\alpha_{j}, \beta_{j}\right]\right)\left[\alpha_{p-1}, \bar{\alpha} \beta_{p-1}\right]= \\
s\left[\alpha_{p-1}, \beta_{p-1}\right]^{-1} \alpha_{p-1} \alpha_{p}\left[\alpha_{p}, \beta_{p}\right]^{-1} \alpha_{p-1}^{-1}\left[\alpha_{p-1}, \beta_{p-1}\right]\left[\alpha_{p}, \beta_{p}\right] \alpha_{p}^{-1} .
\end{gathered}
$$

It is convenient to streamline our notation. We put

$$
g_{1}=\prod_{1}^{p-2}\left[g_{\alpha_{j}}, g_{\beta_{j}}\right], g=g_{\alpha_{p-1}}, h=g_{\beta_{p-1}}, k=g_{\alpha_{p}}, l=g_{\beta_{p}} .
$$

We have $(\sigma \cdot F)(g)=f\left(g_{\sigma \circ s}\right)$. Hence the $\sigma$-invariance of $F$ is equivalent to

$$
f\left(g_{1}[g, h]\right)=f\left(\left(g_{1} g\right)\left(k k_{c}^{-1}\right) g_{1}[g, h]\left(g_{1} g\right)^{-1}\left(k k_{c}^{-1}\right)^{-1}\right),
$$


for a.e. $g_{1}, g, h, k, l$, subject to the constraint $g_{1}[g, h][k, l]=k_{c}$.

Define

$$
\begin{gathered}
\phi:\left\{\left(g_{1}, g, h, k, l\right): g_{1}[g, h][k, l]=k_{c}\right\} \rightarrow K^{\prime} \times K^{\prime} \\
\phi:\left(g_{1}, g, h, k, l\right) \rightarrow\left(g_{1}[g, h],\left(g_{1} g\right)\left(k k_{c}^{-1}\right) g_{1}[g, h]\left(g_{1} g\right)^{-1}\left(k k_{c}^{-1}\right)^{-1}\right) ;
\end{gathered}
$$

(3.8) is equivalent to $\phi_{1}^{*} f=\phi_{2}^{*} f$, a.e.. If this equality held at all points, then to prove that $f$ is constant, it would suffice to show that the relation defined by $\operatorname{Im}(\phi)$ (or the equivalence relation generated by $\operatorname{Im}(\phi)$ ) is transitive; since the equality holds in an a.e. sense, we must consider the relation defined by the interior of $\operatorname{Im}(\phi)$. It is plausible that $\phi$ is surjective, but we can only prove the following weaker result.

Lemma (3.10). Let $p r_{1}: K^{\prime} \times K^{\prime} \rightarrow K^{\prime}:(m, n) \rightarrow m$. Then $K^{\prime} \backslash p r_{1}(\operatorname{Interior}(\operatorname{Im}(\phi)))$ has real codimension at least 2 in $K^{\prime}$.

This Lemma implies that for each $m \in p r_{1}(\operatorname{Interior}(\operatorname{Im}(\phi)))$, we can find open sets $U_{m}$ and $V_{m}$ in $K^{\prime}$ such that $m \in U_{m}$ and $U_{m} \times V_{m} \subset \operatorname{Im}(\phi)$. Since $\phi_{1}^{*} f=$ $\phi_{2}^{*} f$, a.e., it follows that $f$ is constant on $U_{m}$, a.e.. Since $p r_{1}(\operatorname{Interior}(\operatorname{Im}(\phi)))$ is connected, this constant must be the same for each $U_{m}$. This implies that $f$ is constant a.e.. Thus proving (3.10) will complete the proof of (3.1).

Proof of Lemma (3.10). We can suppose that $p=2$, which amounts to setting $g_{1}=1$, and that $K=K^{\prime}$. For notational simplicity we will abbreviate $\operatorname{Ad}(g)(\cdot)$ to $g(\cdot)$.

To prove (3.10), we first claim that it suffices to show that the map

$$
\psi_{m}:\{[g, h]=m\} \times\left\{[k, l]=m^{-1} k_{c}\right\} \rightarrow K:(g, h ; k, l) \rightarrow g k k_{c}^{-1} m g^{-1}\left(k k_{c}^{-1}\right)^{-1}
$$

is regular at some smooth point of its domain, for each $m \in K \backslash Y$, where $Y$ has codimension 2. For if $\psi_{m}$ is regular at the smooth point $(g, h ; k, l)$, then $(g, h)$ is regular for the commutator map, which is the first factor of $\phi$. Thus $\operatorname{Im}\left(\left.d \phi\right|_{(g, h, k, l)}\right)$ spans both the vertical and horizontal directions, hence $(g, h ; k, l)$ is regular for $\phi$.

To specify $Y$, consider the commutator map [,] $: K \times K \rightarrow K$. This map is surjective, the fibers generically have dimension $d=\operatorname{dimn}(K)$, and the exceptional fibers have dimension exceeding $d$ (e.g. [, $]^{-1}(1)$ has dimension $d+r, r=\operatorname{rank}(K)$ ). Let $N$ denote the set of values $n \in K$ such that there exists $(g, h)$ with $[g, h]=n$ and (i) $g \in K^{\text {reg }}$ and (ii) $\mathfrak{k}^{g} \cap \mathfrak{k}^{h}=\{0\}$ (i.e. $(g, h)$ is regular for [,]). By (B.5) of Appendix B, $K \backslash N$ has codimension at least 2 in $K$. We set

$$
K \backslash Y=\left\{m: \quad m \in N \text { and } m^{-1} k_{c} \in N\right\} .
$$

The Zariski tangent space to $[,]^{-1}(m)$ at $(g, h)$ is given by

$$
\left.T\right|_{(g, h)}=\left\{(x, y): x^{h^{-1}}-x+y-y^{g^{-1}}=0\right\} .
$$


Projection onto the $x$ factor induces the exact sequence

$$
\left.0 \rightarrow\left\{(0, y): y \in \mathfrak{k}^{g}\right\} \rightarrow T\right|_{(g, h)} \rightarrow\left\{x:\left(1-h^{-1}\right) x \perp \mathfrak{k}^{g}\right\} \rightarrow 0 .
$$

If $(g, h)$ satisfies (i) and (ii), then $(g, h)$ is a smooth point, and the spaces in $(3.14)$ have dimensions $r, d$, and $d-r$, respectively. Note that

$$
\left\{x:\left(1-h^{-1}\right) x \perp \mathfrak{k}^{g}\right\}=\left((1-h)^{\mathfrak{k}^{g}}\right)^{\perp},
$$

and this space depends only upon $g$ : since $h g h^{-1}=\left(g^{-1} m\right)^{-1}, h$ is unique up to multiplication on the right by $\lambda \in C_{K}(g)$, and $\lambda$ acts trivially on $\mathfrak{k}^{g}$.

Fix $m \in K \backslash Y$. The derivative of the map $\psi_{m}$ is given by

$$
\begin{gathered}
\left.d \psi_{m}\right|_{(g, h ; k, l)}:(x, y ; z, w) \rightarrow\left(x^{m^{-1}\left(k k_{c}^{-1}\right)^{-1}}-x\right)^{k k_{c}^{-1} g}+\left(z^{k_{c}^{-1} g m^{-1} k_{c}}-z\right)^{k} \\
=\left(x^{\left(k k_{c}^{-1} m\right)^{-1}}-x+z^{m^{-1} k_{c}}-\left(z^{m^{-1} k_{c}}\right)^{g^{-1} m}\right)^{k k_{c}^{-1} g} .
\end{gathered}
$$

Together with (3.14) and (3.15) this means that we must show that for suitable $g, h, k, l$, the sum of subspaces

$$
\left(1-\left(k k_{c}^{-1} m\right)^{-1}\right)\left((1-h) \mathfrak{k}^{g}\right)^{\perp}+\left(1-g^{-1} m\right) m^{-1} k_{c}\left((1-l) \mathfrak{k}^{k}\right)^{\perp}
$$

is all of $\mathfrak{k}$.

Now to deal with (3.17), we need some control over solutions to the constraint equations $[g, h]=m,[k, l]=m^{-1} k_{c}$. For this purpose, consider the equation $\left[g_{1}, h_{1}\right]=n$. In (B.1) of Appendix B, we show that for any maximal torus $T$, there exists a solution $\left(g_{1}, h_{1}\right)$ with $g_{1} \in T$. For $n \in N$, by dimensional considerations and the fact that these equations are algebraic, $g_{1}$ is a finite multi-valued function of $T$ (see (a) of (B.6) for explicit equations). Apply this to $n=m$. Given $T$, we obtain solutions $[g, h]=m$. We have $g^{-1} m=h g^{-1} h^{-1} \in h T h^{-1}$. Therefore we obtain a finite number of tori $h T h^{-1}$. We claim that the multi-valued map

$$
\phi_{m}:\{\text { Tori }\} \rightarrow\{\text { Tori }\}: T \rightarrow h T h^{-1} \subset C_{K}\left(g^{-1} m\right)
$$

is surjective. In a loose sense the inverse is $\phi_{m^{-1}}$, because $\left[h g h^{-1}, h^{-1}\right]=m^{-1}$. More precisely, given a torus $T_{1}$, apply the preceding to $m^{-1}$ and $T_{1}$ to obtain $\left(g_{1}, h_{1}\right)$ with $\left[g_{1}, h_{1}\right]=m^{-1}$ and $g_{1} \in T_{1}$. Define $(g, h)$ and $T$ so that $g_{1}=h g h^{-1}$, $h_{1}=h^{-1}, T=h_{1} T_{1} h_{1}^{-1}$. Then $[g, h]=m$, and $T_{1}=h T h^{-1}$. This proves the claim.

Similarly the multi-valued map

$$
\Phi_{m^{-1} k_{c}}:\{\text { Tori }\} \rightarrow\{\text { Tori }\}: T \rightarrow(k l) T(k l)^{-1} \subset C_{K}\left(k k_{c}^{-1} m\right),
$$

where $[k, l]=m^{-1} k_{c}, k \in T$, is surjective, and the inverse, again in a loose sense, is $\Phi_{k_{c}^{-1} m}$. For given $T_{1}$ we can find $\left[k_{1}, l_{1}\right]=k_{c}^{-1} m, k_{1} \in T_{1}$. Define 
$k=\left(k_{1} l_{1}\right) k_{1}\left(k_{1} l_{1}\right)^{-1}, l=\left(k_{1} l_{1}\right) k_{1}^{-1}\left(k_{1} l_{1}\right)^{-2}, T=\left(k_{1} l_{1}\right) T_{1}\left(k_{1} l_{1}\right)^{-1}$. Then $[k, l]=$ $m^{-1} k_{c}, k \in T$, and $T_{1}=k l T(k l)^{-1}$.

Choose the pairs $(g, h)$ and $(k, l)$ such that $[g, h]=m$ and $[k, l]=m^{-1} k_{c}$, and such that both pairs satisfy (i) and (ii) above. It may be necessary to consider perturbations of these pairs. We will refer to perturbations which fix the constraints as admissible. The conditions (i) and (ii) are stable under small admissible perturbations. The space [, $]^{-1}(m)$ has dimension $d$, and for $g$ as above the possible $h$ 's with $[g, h]=m$ form an $r$ dimensional set. Thus an admissible small perturbation of $(g, h)$ gives a smooth $d-r$ dimensional perturbation of $g$, the tangent space of which is described by (3.15). The same comments apply to $(k, l) \in[,]^{-1}\left(m^{-1} k_{c}\right)$.

Now consider the subspace represented by the first term in (3.17). We first fix $g$ and $h$. We claim that we can choose an arbitrarily small admissible perturbation of $(k, l)$ such that

$$
\begin{gathered}
\left(1-\left(k k_{c}^{-1} m\right)^{-1}\right)\left((1-h) \mathfrak{k}^{g}\right)^{\perp} \\
=\operatorname{Im}\left(1-\left(k k_{c}^{-1} m\right)^{-1}\right)=\left(\mathfrak{k}^{k k_{c}^{-1} m}\right)^{\perp} .
\end{gathered}
$$

This will hold if we can arrange for $\left((1-h) \mathfrak{k}^{g}\right)^{\perp}$ to intersect $\operatorname{ker}\left(1-\left(k k_{c}^{-1} m\right)^{-1}\right)$ trivially, i.e.

$$
\mathfrak{k}^{k k_{c}^{-1} m} \cap\left((1-h) \mathfrak{k}^{g}\right)^{\perp}=\{0\} .
$$

Because $k$ is regular, $k k_{c}^{-1} m=(k l) k(k l)^{-1}$ is regular. Thus $\mathfrak{k}^{k k_{c}^{-1} m}$ has dimension $r$, and $\left((1-h)^{\mathfrak{k}^{g}}\right)^{\perp}$ has dimension $d-r$ (the latter statement is equivalent to condition (ii)). By (B.1) and (3.19) we can find an arbitrarily small admissible perturbation of $(k, l)$ such that the intersection (3.21) will be zero.

We now fix our choice of $(k, l)$. We claim that we can find an arbitrarily small admissible perturbation of $(g, h)$ such that

$$
\left(1-g^{-1} m\right) m^{-1} k_{c}\left((1-l) \mathfrak{k}^{k}\right)^{\perp}=\left(\mathfrak{k}^{g^{-1} m}\right)^{\perp} .
$$

The argument is essentially the same. It suffices to establish

$$
\mathfrak{k}^{g^{-1} m} \cap m^{-1} k_{c}\left((1-l) \mathfrak{k}^{k}\right)^{\perp}=\{0\} .
$$

As before, $g^{-1} m$ is regular, because $g$ is regular. By (3.18) we can arrange this by an arbitrarily small admissible perturbation.

We now have found $g, h, k, l$ such that the image of the subspace (3.17) equals

$$
\left(\mathfrak{k}^{k k_{c}^{-1} m}\right)^{\perp}+\left(\mathfrak{k}^{g^{-1} m}\right)^{\perp}=\left(\mathfrak{k}^{k k_{c}^{-1} m} \cap \mathfrak{k}^{g^{-1} m}\right)^{\perp},
$$

and this equality is stable under small admissible perturbations. Again by (3.18) and (3.19) we can find a small admissible perturbation so that (3.24) will be all of $\mathfrak{k}$.

We have now proven that the map $\psi_{m}$ is regular at some smooth point for each $m \in K \backslash Y$, and as we observed at the beginning of the proof, this implies (3.10). 


\section{Appendix A. Connectedness properties}

The following results can be deduced from [BR] (and perhaps elsewhere). We record them here for the convenience of the reader.

Lemma (A.1). Suppose that $K$ is simply connected. Then $\{(g, h) \in K \times K$ : $[g, h]=k\}$ is connected, $\forall k \in K$.

If $C$ denotes the conjugacy class containing $k$, then there is a surjective map

$$
\{[g, h]=k\} \rightarrow\{[g, h] \in C\} / \operatorname{conj}(K)
$$

and the fibers are homogeneous spaces for $K$. The fibers are connected because $K$ is connected, and by [BR] the moduli space corresponding to $C$ is connected because $K$ is simply connected. This establishes (A.1) (It would clearly be desirable to give an elementary direct proof of this.)

Lemma (A.2). Suppose that $K$ is simply connected. Suppose that $\Sigma$ is an object with group element boundary condition which is obtained by sewing one-holed tori to an $N$-holed sphere. Then $\operatorname{Hom}(\Sigma, K)$ is connected.

Proof. The space Hom for an $N$-holed sphere is empty or a point. When we sew, we obtain a connected object by (1.3).

Let $p r: \tilde{K} \rightarrow K$ denote the universal covering of $K$.

Proposition (A.3). If $\Sigma$ is a one-holed surface with boundary condition $l \in K$, then we have the decomposition into connected components

$$
\operatorname{Hom}\left(\Sigma_{l}, K\right)=\bigsqcup_{\tilde{l} \in \tilde{K}^{\prime} \cap p r^{-1}(l)} p r_{*} \operatorname{Hom}\left(\Sigma_{\tilde{l}}, \tilde{K}\right) .
$$

This decomposition is equivariant with respect to $\pi_{0}(\operatorname{Aut}(\Sigma))$.

This follows from (A.2).

\section{Appendix B. Commutators}

At several points of this paper, we used the fact that the commutator map [,] : $K \times K \rightarrow K^{\prime}$ is surjective (and we presented an indirect proof of this in (a) of (2.1.5)). Here we discuss some refinements which we use in the proof of (3.10).

Proposition (B.1). Let $T$ denote a maximal torus in $K$. The map

$$
\psi: T \times K \rightarrow K^{\prime}:(\lambda, h) \rightarrow[\lambda, h]
$$

is surjective. 
Proof of Proposition (B.1). To simplify the notation, we will write $K$ in place of $K^{\prime} ; d$ will denote the dimension, and $r$ the rank, of $\mathfrak{k}$.

The derivative of $\psi$ at $(\lambda, h)$ is given by

$$
\mathfrak{t} \times \mathfrak{k} \rightarrow \mathfrak{k}:(x, y) \rightarrow\left(x^{\lambda h^{-1}}-x^{\lambda}+y^{\lambda}-y\right)^{h},
$$

hence the image of the derivative at $(\lambda, h)$ is

$$
\begin{gathered}
\operatorname{Ad}(h)\left(\operatorname{Ad}(\lambda)\left(\left(1-\operatorname{Ad}\left(h^{-1}\right)\right) \mathfrak{t}\right)+\left(\mathfrak{k}^{\lambda}\right)^{\perp}\right) \\
=\operatorname{Ad}(h \lambda)\left(\left(1-\operatorname{Ad}\left(h^{-1}\right)\right) \mathfrak{t}+\left(\mathfrak{k}^{\lambda}\right)^{\perp}\right) .
\end{gathered}
$$

We claim that the point $(\lambda, h)$ is critical for $\psi$ if and only if (i) $\lambda \notin K^{\text {reg }}$ or (ii) $\mathfrak{k}^{h} \cap \mathfrak{t} \neq\{0\}$. To see this, suppose that $\lambda$ is regular and $\mathfrak{k}^{h} \cap \mathfrak{t}=\{0\}$. Then $\left(\mathfrak{k}^{\lambda}\right)^{\perp}=$ $\mathfrak{t}^{\perp}$ has dimension $d-r$ and $\left(1-\operatorname{Ad}\left(h^{-1}\right)\right) \mathfrak{t}$ has dimension $r$. If the intersection of these two spaces is nonempty, then there is $x \in \mathfrak{t}$ such that $x^{h^{-1}}=x+y^{\perp}$, where $y^{\perp} \in \mathfrak{t}^{\perp}$ is not zero; but $x^{h^{-1}}$ and $x$ have the same length, so that $x \perp y^{\perp}$ implies $y^{\perp}=0$, which is a contradiction. Thus the dimension of the space (B.3) is $d$, and this establishes our claim.

We can factor $\psi=\tilde{\psi} \circ p$, where

$$
T \times K \stackrel{p}{\rightarrow} T \times K / T \stackrel{\tilde{\psi}}{\longrightarrow} K:(\lambda, h) \stackrel{p}{\rightarrow}(\lambda, h T) \stackrel{\tilde{\psi}}{\rightarrow}[\lambda, h],
$$

so that at any regular point, $\tilde{\psi}$ will actually be a local diffeomorphism. We claim that the set of critical values for $\tilde{\psi}$ has codimension at least two. This will imply that $\tilde{\psi}$ is surjective, because a boundary for the image would necessarily have codimension one.

Suppose that (i) holds, i.e. $\lambda_{0} \notin T^{\text {reg. }}$. In this case, as we vary $h, \lambda_{0} h \lambda_{0}^{-1} h^{-1}$ will sweep out the $\lambda_{0}$-translate of a nongeneric conjugacy class, which will have dimension $\leq d-r-2$. Thus the dimension of the set of critical values arising from condition (i) will be $\leq r-1+d-r-2=d-3$.

Now suppose that (ii) holds. The subset $\left\{h_{0} \in K: \mathfrak{k}^{h_{0}} \cap \mathfrak{t} \neq 0\right\}$ has codimension at least 2 in $K$ : if $h_{0}=\exp (X)$, where $X$ is regular, then we must have $X_{\alpha}=0$ for some root $\alpha$ of $\mathfrak{t}$, where $X_{\alpha}$ denotes the $\alpha$-root space component of $X$ (see the proof of (2.1.24), especially the paragraph containing (2.1.28)). This is a $T$-invariant condition, hence the set of critical points corresponding to (ii) has codimension at least 2 in $K / T$. It follows that the corresponding set of critical values has dimension $\leq r+d-r-2$. This completes the proof.

Corollary (B.5). For the commutator map [,] : $K \times K \rightarrow K^{\prime}$, the complement of the subset $N$ of $K^{\prime}$ defined by

$$
\left\{n: \exists(g, h) \in[,]^{-1}(n) \quad \text { s.t. } \quad \text { (i) } g \in K^{\mathrm{reg}}, \quad \text { (ii) } \mathfrak{k}^{g} \cap \mathfrak{k}^{h}=\{0\}\right\}
$$

has codimension at least 2. 
Proof. Given a maximal torus $T$, each regular value for the map $\psi$ of (B.1) will belong to $N$. In the proof of (B.1) we established that the complement of the set of regular values for $\psi$ has codimension at least 2. By varying $T$, we obtain (B.5).

Remarks (B.6). (a) It is of interest to consider the more general question of whether, for given $g \in K$, the map

$$
\psi_{g}: T \times K^{\prime} \rightarrow K^{\prime}:(\lambda, h) \rightarrow[g \lambda, h]
$$

is surjective. This has a factorization

$$
\begin{gathered}
T \times K \stackrel{p}{\rightarrow} D_{g}=\{(\lambda, l) \in T \times K: g \lambda \sim l\} \stackrel{\tilde{\psi}_{g}}{\longrightarrow} K \stackrel{L_{g}}{\longrightarrow} K \\
(\lambda, h) \stackrel{p}{\longrightarrow}\left(\lambda, h g \lambda h^{-1}\right)=(\lambda, l) \stackrel{\tilde{\psi}_{g}}{\longrightarrow} \lambda l^{-1}=k \stackrel{L_{g}}{\longrightarrow} g k,
\end{gathered}
$$

where $g \lambda \sim l$ means $g \lambda$ and $l$ are conjugate. The map $\tilde{\psi}_{g}$ is the restriction to $D_{g}$ of the natural coset fibration

$$
T \times K \rightarrow(T \times K) / \Delta(T),
$$

where $\Delta(T)$ is the diagonally embedded copy of $T$ in $T \times K$, and we identify $(T \times K) / \Delta(T)$ with $K$ by $(\lambda, l) \Delta(T) \leftrightarrow \lambda l^{-1}$. The map $\tilde{\psi}_{g}$ is surjective if and only if for each $k \in K$, there exists $\lambda \in T$ such that $g \lambda \sim l=k^{-1} \lambda$. This is equivalent to a system of $r$ polynomial equations

$$
\chi_{i}(g \lambda)=\chi_{i}\left(k^{-1} \lambda\right), \quad i=1, \ldots, r
$$

for $r$ unknowns $\lambda_{1}, \ldots, \lambda_{r} \in \mathbb{T}$, where $\chi_{i}$ is the character corresponding to the $i^{\text {th }}$ fundamental irreducible representation, and $\lambda=\prod_{1}^{r} \lambda_{i}^{h_{i}}$, where the $h_{i}$ are the coroots (e.g. for $S U(3)$, we have 2 equations

$$
\sum_{1}^{3} A_{i} \lambda_{i}=0, \quad \bar{A}_{1} \lambda_{2} \lambda_{3}+\bar{A}_{2} \lambda_{1} \lambda_{3}+\bar{A}_{3} \lambda_{1} \lambda_{2}=0
$$

for the $\lambda_{i} \in \mathbb{T}$, subject to the constraint $\prod \lambda_{i}=1$, where $\left.A_{i}=g_{i i}-\left(k^{-1}\right)_{i i}\right)$. It is trivial to check that for $S U(2), \psi_{g}$ is always surjective, but this is not so for $S U(3)$. Thus in particular the equations (B.11) do not in general have solutions satisfying the reality condition $\left|\lambda_{i}\right|=1$; on the other hand (B.1) asserts that such solutions always exist for $g=1$.

This suggests a number of questions, such as how does one describe the set of conjugacy classes which meet $g T$, when is $\psi_{g}$ surjective, and so on. 
(b) Identify $S U(2)$ with $\mathbb{H}_{1}$, the group of unit quaternions, by $\left(\begin{array}{cc}a & b \\ -\bar{b} & \bar{a}\end{array}\right) \leftrightarrow$ $q=a-b j$, and take $T=\mathbb{T}$. The conjugacy classes in $\mathbb{H}_{1}$ are obtained by fixing the real part of $q$. Now fix $g=a-b j$. The conjugacy classes which meet $g \mathbb{T}$ are indexed by $[-|a|,|a|]$. We have

$$
\begin{gathered}
D_{g}=\left\{(\lambda, q) \in \mathbb{T} \times \mathbb{H}_{1}: \mathbb{R} e(q)=\mathbb{R} e(a \lambda)\right\}, \\
\tilde{\psi}_{g}: D_{g} \rightarrow \mathbb{H}_{1}:(\lambda, q) \rightarrow \lambda \bar{q}, \\
\left.T D_{g}\right|_{\lambda, q}=\left\{\left(i s, q^{\prime}\right) \in i \mathbb{R} \times \operatorname{Im}(\mathbb{H}): \mathbb{R} e(a \lambda i s)=\mathbb{R} e\left(q q^{\prime}\right)\right\}, \\
d\left(\tilde{\psi}_{g}\right):\left.T D_{g}\right|_{\lambda, q} \rightarrow \operatorname{Im}(\mathbb{H}):\left(i s, q^{\prime}\right) \rightarrow q\left(i s+\bar{q}^{\prime}\right) \bar{q}, \\
D_{g, \text { critical }}=\left\{\left(\lambda, a \lambda+\sqrt{1-|a|^{2}} z j\right): \lambda, z \in \mathbb{T}\right\} .
\end{gathered}
$$

When $0<|a|<1$, the singular set is a 2 -torus; at the extreme values $|a|=0,1$, the critical set degenerates to a circle. The $S U(2)$ miracle is that in all cases, the set of critical values

$$
\tilde{\psi}_{g}\left(D_{g, \text { critical }}\right)=\left\{\bar{a}-\sqrt{1-|a|^{2}} \lambda z j: \lambda, z \in \mathbb{T}\right\}
$$

is a circle. One can easily visualize how $\tilde{\psi}_{g}$ covers $\mathbb{H}_{1}$.

The extreme case $|a|=0$, when there is just a single (totally geodesic) conjugacy class, corresponds to the condition that $g$ is a so-called principal element $([\mathrm{K}])$.

\section{References}

[AB] M. Atiyah and R. Bott, The Yang-Mills equations over Riemann surfaces, Phil. Trans. R. Soc. Lond. A308 (1982), 523-615.

[BR] U. Bhosle and A. Ramanathan, Moduli spaces of principal bundles with parabolic structure over Riemann surfaces, Math. Z. 202 (1989), 161-180.

[Bt] T. Bröcker and T. tom Dieck, Representations of Compact Lie Groups, Springer-Verlag, 1995.

[DMS] P. Di Francesco, P. Mathieu and D. Senechal, Conformal Field Theory, Springer-Verlag, 1996.

[Fo] R. Forman, Small volume limits of 2D Yang-Mills, Comm. Math. Physics 151 (1993), 39-52.

[Go1] W. Goldman, The symplectic nature of fundamental groups of surfaces, Adv. Math. 54 (1984), 200-225.

[Go2] W. Goldman, Ergodic theory on moduli spaces, Ann. Math. 146 (1997), 475-507.

$[\mathrm{K}] \quad$ B. Kostant, The principal three-dimensional subgroups and the Betti numbers of a complex simple Lie group, Amer. J. Math. 81 (1959), 973-1032.

[Pi] D. Pickrell, On $Y M_{2}$ measures and area-preserving diffeomorphisms, J. Geom. Phys. 19 (1996), 315-367. 
[PX] J. P. Previte and E. Z. Xia, Topological dynamics on moduli spaces, I, Pac. J. Math. 193, No. 2 (2000), 397-417; II, arXiv:math.DS/99100036.

[V] V. S. Varadarajan, Lie Groups, Lie Algebras, and Their Representations, Springer-Verlag. 1984.

Doug Pickrell

University of Arizona

Department of Mathematics

Tucson, AZ 85721

USA

e-mail: pickrell@math.arizona.edu

(Received: March 21, 2001)
Eugene Z. Xia

University of Massachusetts

Department of Mathematics and Statistics

Amherst, MA 01003-4515

USA

e-mail: xia@math.umass.edu

(20) To access this journal online:

(20) http://www.birkhauser.ch 